\title{
La didactique du FLM, du FLE et du plurilinguisme au service de l'éveil aux styles à l'école : des pistes pour la formation des enseignants
}

\author{
Laurence Buson \\ Laboratoire Lidilem - Université Stendhal Grenoble3 \\ laurence.buson@u-grenoble3.fr
}

Si la didactique de l'oral fait aujourd'hui l'objet de nombreuses recherches, la question de la variation stylistique et de l'enseignement/apprentissage des styles/registres de langue ${ }^{1}$ est encore peu interrogée. Pourtant, savoir s'adapter à la situation de communication et avoir pris conscience des rapports complexes entre sens social et usages langagiers en contexte relève d'un faisceau de compétences complexe que l'école se doit d'investir. Favoriser l'émergence d'une conscience métapragmatique et de répertoires communicatifs larges et diversifiés semble en effet constituer une mission de toute première importance pour former des élèves citoyens capables d'évoluer dans des univers langagiers variés tels que la famille, le réseau de pairs, l'école, ou encore, par la suite, le monde professionnel.

Afin d'explorer les enjeux relatifs à cette problématique et de fournir, pour la formation des enseignants, des pistes que nous souhaitons ancrées dans la réalité des pratiques existantes et néanmoins fondées sur les recherches interdisciplinaires actuelles, nous nous intéresserons à la fois aux programmes officiels, généralement peu prolixes sur la dimension variationnelle de la langue, et aux modalités d'entrée dans la notion de registre de langue de quelques manuels de français récents. Nous discuterons ensuite certaines orientations didactiques allant dans le sens de ce que l'on pourrait qualifier d'éveil aux styles ${ }^{2}$.

\section{Les Instructions Officielles et les manuels : quelles ressources les enseignants peuvent-il exploiter?}

Encore aujourd'hui, seul le lexique est concerné lorsqu'il s'agit de faire des recommandations sur l'enseignement de la variation stylistique, et cette question est réduite à la portion congrue dans les derniers programmes pour le cycle 3 :

\footnotetext{
Une première réflexion sur le lexique /.../ permet aussi de commencer à distinguer le rôle joué par le lexique dans le choix d'un registre de langue. (M.E.N., 2002: 75)

\begin{abstract}
Dans des situations d'échanges variées, il [l'élève] apprend à tenir compte des points de vue des autres, à utiliser un vocabulaire précis appartenant au niveau de la langue courante, à adapter ses propos en fonction de ses interlocuteurs et de ses objectifs. (M.E.N., 2008: 21)
\end{abstract}

Prendre la parole en respectant le "niveau de langue adapté" fait donc partie des compétences attendues en fin de CM2, sans qu'il ne soit fait état d'une volonté réelle de prise en compte de la variation. L'objectif affiché ici est que l'élève sache s'exprimer d'une manière acceptable en classe, et aucune approche réflexive sur le style n'est encouragée. Les prémisses de ce travail concernent le CM1, où l'enfant peut "commencer à identifier les différents niveaux de langue", entre autres par l'usage du dictionnaire, dans le cadre d'un travail sur la "maitrise du sens des mots" (M.E.N., 2008: 35).

Il est de plus remarquable de constater que la prise en compte des caractéristiques de l'oral est rudimentaire, avec une référence claire aux unités de l'écrit :

$\mathrm{Au}$ cycle des apprentissages fondamentaux, les élèves continuent leur apprentissage du langage oral : respect de l'organisation de la phrase, expression des relations de causalité et des circonstances temporelles et spatiales (pourquoi ? quand ? où ?) ; utilisation plus adéquate de la conjugaison, emploi d'un vocabulaire de plus en plus 
diversifié ; prises de parole de plus en plus longues et mieux organisées, dans le respect des sujets traités et des règles de la communication. (M.E.N., 2008: 17)

Ce postulat de règles régissant la communication qu'il s'agirait de respecter n'est néanmoins pas davantage explicité. L'accent est mis prioritairement sur la qualité, la correction et la précision de l'expression (M.E.N., 2008: 22) et sur la longueur des prises de parole, ce qui peut laisser perplexe, d'autant plus que l'étalon d'évaluation de ce critère semble être la "phrase orale". En définitive, les objectifs sont vagues, peu ambitieux et les mises en œuvre sont laissées à la discrétion des enseignants.

De la même manière, dans les manuels même récents ${ }^{3}$, qu'il s'agisse du primaire ou du collège, la notion de registre de langue est presque toujours abordée dans la confusion la plus totale, tant au niveau de l'association entre oral, familier, et incorrect, de l'association entre familier et variété diatopique/diastratique, et des jugements de valeur sur les variétés et les locuteurs. Les typologies proposées, toujours maladroites et imprécises, souvent erronées, aboutissent à des incohérences flagrantes, comme par exemple, dans Les Couleurs du Francais 6ème (Himy, Artaud et al., 2009), le fait d'affirmer, sur une même page, que le niveau familier, qualifié de "négligé", correspond au langage oral, avant d'illustrer cette notion avec la phrase "Le géant se cassa avec la meuf". Il est, en effet, improbable que les élèves comprennent et s'approprient un classement qui sous-entend que l'emploi du passé simple dans un énoncé, qui plus est sans doublement du sujet, est un bon exemple de langue orale "relâchée".

De plus, si les sempiternelles activités de traduction restent très répandues dès qu'il s'agit d'aborder la notion de registre de langue (Boutet \& Gadet, 2003; Gadet \& Tyne, 2007: 92), et si elles portent essentiellement sur le vocabulaire, c'est probablement pour éviter de se confronter à l'impossibilité d'appréhender le phénomène de la variation stylistique dans toute sa complexité. En effet, si l'on tente d'appliquer cette gymnastique d'établissement d'équivalences lexicales à des énoncés du quotidien même très simples, les limites apparaissent très vite : peut-on par exemple dire que le "comment ça va ?" adressé à un ami se traduira par "comment cela va-t-il ?"4 à un individu moins familier ? Si cette dernière formulation n'est pas fausse au regard de la norme syntaxique, elle n'en est pas moins étrange au regard de la norme objective, qui préférera sans doute un "comment allez-vous ?", voire un "vous allez bien ?". La manipulation simpliste de certains traits stylistiques ponctuels ne permet donc pas de discuter des différents sens véhiculés par l'énoncé dans sa globalité, et ne semble pas répondre aux objectifs, même vagues, fixés par les programmes, de savoir adapter sa manière de parler aux situations et aux interlocuteurs.

Malgré ces constats quelque peu négatifs, il est à noter que certains manuels, relativement rares, tentent d'intégrer la variation de façon plus nuancée dans les activités qu'ils proposent. Par exemple, Français $5^{\text {ème }}$ (Oriol-Boyer, Duminy-Sauzeau et al., 1995) aborde la question des registres de langue en ne se restreignant pas au lexique. À partir de la comparaison de deux traductions différentes d'un extrait de De l'autre côté $d u$ miroir de Lewis Carroll ${ }^{5}$, les élèves sont amenés à observer les variations susceptibles d'imprimer un ton plus ou moins familier au récit. Les éléments sur lesquels l'accent est mis concernent le lexique, mais aussi le tutoiement/vouvoiement, et le système des temps. La recherche de traits divers sur lesquels peut porter la variation nous paraît être une entrée intéressante pour aborder la question du style, même si la terminologie utilisée reste très traditionnelle: langage courant ou familier vs langage recherché ou soutenu. La définition des deux registres fournie plus loin dans le manuel (1995: 199) ne permet, en effet, pas de réfléchir à la question du continuum stylistique, et aborde la notion d'une manière étonnamment normative, en laissant de côté les différences entre l'oral et l'écrit: le vocabulaire et la syntaxe corrects sont ainsi censés caractériser le registre soutenu, tandis que le vocabulaire "imagé" et la syntaxe "moins correcte" doivent permettre de reconnaître le registre familier.

Au-delà de ces raccourcis qui pourraient faire l'objet de davantage de nuances, on peut noter que la question des effets de sens inhérents aux variations de style est abordée, ce qui est rarement le cas dans les manuels. Les différents registres et le projet/l'intention des traducteurs sont en effet mis en perspective : le manuel établit clairement un lien entre le registre utilisé et ce que peut ressentir le lecteur, un registre familier dans un dialogue imprimant un côté plus bienveillant au personnage, et un registre recherché un côté plus prétentieux. Dans cette optique, le style n'est plus que décoratif, il connote le texte et participe à la construction du sens. 
Notons que l'on n'échappe pas à l'exercice traditionnel de traduction d'un style dans un autre mais, la tâche portant sur un extrait de roman et non sur des phrases fabriquées ou des mots isolés, il est possible d'envisager un travail réflexif pertinent à partir des différentes productions des élèves. La discussion qui pourra être menée ne se bornera alors pas à fournir une solution, mais bel et bien un ensemble de possibles stylistiques portant sur tous les niveaux linguistiques, en réfléchissant aussi aux situations qui lui donnent sens.

Dans ce même manuel, la problématique de l'hétérogénéité des styles est évoquée, apparemment non pas pour inciter les élèves à réfléchir sur cette caractéristique, mais pour les encourager à produire des écrits stylistiquement homogènes. L'hétérogénéité constitutive du style est alors abordée sous un angle caricatural, comme un écueil rédactionnel à éviter. Le manuel précise en effet que, pour écrire un texte, "on doit choisir, selon le projet, parmi les trois registres de langue existants" (Oriol-Boyer, DuminySauzeau et al., 1995: 240). Malgré les limites de cet angle de vue, le potentiel du support choisi est intéressant ${ }^{6}$ et peut être utile pour conduire une discussion plus nuancée sur la complexité des registres : l'extrait littéraire proposé transcrit en effet une rédaction d'élève dans laquelle les hiatus stylistiques peuvent servir de base à une réflexion de type métalinguistique. Ainsi, la phrase "il nous énerva tellement que nous lui sautâmes dessus et le foutûmes en dehors du terrain" (Charles, 1990, cité par Oriol-Boyer, Duminy-Sauzeau et al., 1995: 234) peut constituer un point de départ pertinent pour amener les élèves à prendre conscience que le style est un tout qui ne se réduit pas à l'accumulation parfois contradictoire de certains traits. Ici, le lexique et le système des temps ne co-varient pas vers le même pôle du continuum, ce qui crée un effet humoristique.

La prise en compte, dans les approches sur les registres, de la complexité du rapport entre les marques linguistiques utilisées et la dimension symbolique du message ainsi constitué est à rapprocher des propos de Maurer (2001), qui insiste sur le fait que le registre "objectivé" ne peut présumer à lui seul du sens qui se crée dans l'interaction. Le raccourci entre manières de parler familières, populaires, et grossières, ou encore entre manières de parler soutenues et gentilles peut ainsi être interrogé par le biais de différentes formulations jouant sur le hiatus entre style et intention communicative, ou encore style et posture énonciative, comme c'était le cas dans le texte de François Charles cité plus haut.

L'illusion selon laquelle le registre soutenu serait plus policé et moins agressif que le registre familier peut alors être remise en question et discutée avec les élèves, comme le souligne Maurer :

[....] le fait qu'il y ait ou non agressivité n'est pas lié au choix du registre : un très soutenu 'Ta proposition est complètement absurde, comme à l'accoutumée, du reste' sera au moins autant agressif que le familier 'Ca va pas, non ?'. En revanche, le 'Ça craint' des adolescents, pour familier qu'il soit, évite l'expression d'un jugement sur l'autre et est une façon peu agressive de refuser une proposition. (Maurer, 2001: 48)

Certains manuels, mais aussi les œuvres de la littérature de jeunesse ${ }^{7}$, peuvent donc fournir des points de départ judicieux pour les échanges métalinguistiques en classe, et aider ainsi les enseignants à initier le débat. Notre propos, dans le cadre de l'exploration rapide de ces quelques ressources, est en effet de montrer que, si les manuels proposent encore trop souvent une vision réductrice de la variation stylistique, ils peuvent aussi, pour peu qu'on les utilise à bon escient et avec une certaine dose d'esprit critique, constituer des supports efficaces pour une démarche réflexive en classe, au cours de laquelle le parallèle entre l'écrit et l'oral peut être interrogé.

Les enseignants peuvent parfois être rebutés par les trésors de temps et d'imagination que nécessite un travail sur l'oral en l'absence de ressources pédagogiques pré-existantes du fait du caractère très coûteux de la construction de matériaux audios ou vidéos authentiques et adaptés. Le recours à des manuels et à des supports écrits peut alors constituer une aide précieuse et pas nécessairement périlleuse tant que l'enseignant est conscient des spécificités de l'oral par rapport à l'écrit, et que l'échange en classe n'est pas fondé sur une comparaison dévalorisante de l'un par rapport à l'autre.

Dans cette même perspective qui consiste à envisager les ressources existantes afin de ne pas décourager les enseignants par la création de trop nombreuses ressources ex nihilo, les méthodes de Français Langue 
Étrangère peuvent apporter des éléments pertinents pour la mise en place d'activités autour de la variation stylistique en classe.

\section{S'inspirer des ressources du Français Langue Étrangère ?}

En Français Langue Étrangère ou Seconde (désormais FLE/2) comme en français L1, le style a longtemps été abordé plutôt tardivement (au cycle 3 en France, et chez les niveaux avancés en L2), comme s'il s'agissait de l'étape ultime voire optionnelle d'une progression. Aujourd'hui, cette tendance a évolué en FLE/2, mais pas réellement en français L1, où les concepteurs des programmes, suivis des rédacteurs de manuels, ne semblent pas considérer qu'une réelle approche réflexive sur le style puisse être menée au niveau de l'école primaire.

Les recherches portant sur l'appropriation de la variation sociolinguistique en FLE/2 considèrent maintenant le style comme "le témoin, positif, d'une compétence sociolinguistique des apprenants" en cours de construction (Gadet \& Tyne, 2007: 96). Pour évaluer les compétences des locuteurs non natifs, Mougeon, Nadasdi et al. (2002) mettent l'accent sur certaines formes spécifiques, supposées plus ou moins emblématiques des variations situationnelles en français, comme le choix des pronoms on/nous et tu/vous, ou encore le ne de négation. Ils observent alors que les compétences stylistiques des apprenants, même avancés, sont limitées, ces derniers utilisant significativement moins de formes non-standard que les natifs ${ }^{8}$. D'après eux, l'une des explications à ce constat provient d'un input variationnel très réduit, avec un environnement d'apprentissage très normatif :

[...] on a vu que le français des enseignants et des auteurs de manuels offrait aux apprenants un modèle linguistique fortement standardisé, qui ne permet guère à ces derniers d'acquérir un répertoire sociolinguistique proche de celui des locuteurs de FL1. (Mougeon, Nadasdi et al., 2002: 43)

Malgré ces obstacles, il est intéressant de noter que les préoccupations communicatives des méthodes de $\mathrm{FLE} / 2$ et les recherches en didactique dans ce domaine (voir par exemple le recueil d'articles édité par Coleman \& Crawshaw, 1994) ont contribué à accroître la prise en compte de la variation dans certains manuels récents, avec des démarches qui peuvent être qualifiées de plurinormalistes ${ }^{9}$, même si elles sont encore tâtonnantes. La critique des manuels de FLE, si elle reste fondée, mérite donc d'être nuancée en comparaison de ce que proposent actuellement les manuels de français pour les natifs.

Ainsi, par exemple, Alter Ego niveau A2 ${ }^{10}$ (2006: 32-33) aborde la notion de registre de langue en s'appuyant sur un matériau audio fabriqué qui met en scène une simulation d'entretien d'embauche. Les apprenants doivent d'abord essayer d'identifier des différences entre les manières de parler des deux interlocuteurs, sachant que le document est élaboré de manière à souligner le contraste important entre le style du recruteur, très formel, et le style du candidat, très détendu, et peu adapté à la situation. La terminologie est ensuite introduite, avec deux catégories seulement : le standard et le familier. Le manuel propose également une activité de discrimination auditive au cours de laquelle il s'agit de classer des extraits audio dans une grille opposant les deux registres vus précédemment.

Cette activité, bien que sujette à améliorations, comporte à notre sens plusieurs qualités que la plupart des manuels de français L1 ne possèdent pas :

- elle s'appuie sur un discours oral (même s'il n'est pas authentique), ce qui suppose un large éventail de traits à observer, à tous les niveaux linguistiques et pas seulement le niveau lexical,

- elle laisse une place importante à l'écoute et à l'analyse sans a priori de l'extrait, ce qui peut permettre d'engager une discussion intéressante sur les marques que les élèves sont capables de repérer,

- et elle réduit le nombre de registres à deux au lieu de trois. Ce choix nous paraît bien plus satisfaisant que la traditionnelle catégorisation en trois niveaux, toujours implicitement chargée d'idéologie. Ici, la séparation de type standard/non-standard laisse la possibilité d'affiner les observations en glissant éventuellement, au fil des analyses des apprenants et des traits qu'ils sont capables de relever, d'une conception dichotomique du style à une conception fondée sur l'idée d'un continuum à deux pôles. 
La méthode Ici niveau A2 (2007: 66-67) propose également cette même séparation en deux registres seulement. Si des exercices de transposition d'un style à un autre sont à nouveau proposés, il est à noter qu'ils interviennent après une phase de jeux de rôles mise en place en amont, et que le registre familier fait l'objet d'une description sommaire et néanmoins relativement large qui aborde différents niveaux linguistiques : le lexique, la suppression du ne de négation, les contractions de syllabes ("y'a foule !"), l'utilisation du "on" inclusif ("on fait quoi ?"), et les variations articulatoires ("chépa").

Même si ces approches restent simplificatrices au regard de la complexité du phénomène de variation stylistique, il nous paraît intéressant de constater qu'il est possible d'entrer dans la notion par une observation réfléchie de l'oral ${ }^{11}$, grâce à des mises en situation par exemple, et dans une optique à la fois plus descriptive et moins prescriptive.

Il ressort de ce tour d'horizon rapide qu'il serait nécessaire de fournir aux enseignants des outils utilisables et pertinents mais aussi, et peut-être surtout, de leur apporter en amont des éléments de réflexion sur les représentations et les pratiques des enfants, afin qu'ils soient mieux à même, d'une part, d'exploiter au mieux les ressources existantes et, d'autre part, de concevoir des séquences adaptées aux élèves, réellement susceptibles de faire évoluer leurs représentations et de les aider à construire de nouvelles compétences.

\section{Favoriser une approche didactique plurinormaliste et le développement des compétences métapragmatiques : vers un éveil au(x) style(s)}

Une formation des enseignants axée sur la didactique de l'oral ${ }^{12}$ et la variation stylistique à l'école ne peut faire l'économie d'une réflexion sur les représentations et pratiques langagières des élèves, dans et hors de la classe, voire dans et hors de l'école (Boutet, 2002). La violence symbolique inhérente à la dévalorisation des pratiques vernaculaires, comme prolongement naturel à la dévalorisation des pratiques orales et non-standard, est à la fois humainement douloureuse et pédagogiquement inefficace. Le risque est bel et bien de laisser certains enfants s'enfermer dans des représentations dichotomiques et bien étanches du type eux/nous, avec un eux renvoyant aux styles scolaires, et un nous renvoyant aux styles groupaux et/ou familiaux.

Considérer l'enfant comme une "table rase" va à l'encontre de l'évidence pédagogique selon laquelle le point de départ à la construction de nouvelles connaissances langagières doit être l'expérience linguistique et communicative des enfants (Dannequin, 1977). Interroger les représentations des élèves et prendre en compte le milieu socio-scolaire dans lequel ils évoluent sont deux incontournables pour une didactique contextualisée (Blanchet, Moore et al., 2008). Dans le cadre de l'enseignement/apprentissage de la variation stylistique, prendre en compte l'expérience et le vécu langagier et métalangagier des élèves nécessite de mieux connaitre comment ceux-ci pensent le style et comment ils le pratiquent, et d'aborder la notion dans une optique plurinormaliste nécessaire à l'objectivation des pratiques stylistiques ordinaires (Verhoeven, 2000 [1997]).

Or, les compétences métapragmatiques des enfants s'observent chez la majorité des enfants à partir de 7/8 ans (alors qu'elles sont plus rares chez les enfants plus jeunes) et, à partir de 8/9 ans, les enfants se montrent très sensibles à des critères relevant des conventions sociales et de la situation de communication (Buson, 2009). Il existe donc un décalage manifeste entre l'indigence des activités proposées dans les manuels et le potentiel des enfants que nous avons pu observer. Alors que les élèves sont capables de commentaires métalinguistiques très pointus et reposant sur des critères variés envisageant des enjeux symboliques complexes, les manuels les incitent à manipuler des formes dont ils connaissent en général déjà la valeur ${ }^{13}$, comme simple prétexte à l'introduction d'une terminologie qui plus est infondée.

En réalité, plutôt que l'introduction d'une terminologie à partir d'exercices de traduction d'un registre à un autre, il s'agirait de faire évoluer l'enfant d'un maniement inconscient ou semi-conscient vers une maitrise raisonnée des styles, comme le souligne Blanchet : 
La topique contextique conduit ainsi à une didactique 'plurinormaliste', c'est-à-dire métapragmatique /.../. Plusieurs travaux ont récemment confirmé que c'est en passant de l'étude ouverte du système et de ses variations à l'étude d'une norme que celle-ci est la mieux intériorisée, et que les compétences communicatives générales sont les mieux développées. (Blanchet, 1995: 119-120)

De plus, contrairement à certaines idées reçues, les enfants sont souvent, si on les invite à s'exprimer, de bons vecteurs pour la transmission des normes scolaires (Liogier, 2009) : ils sont par exemple conscients des normes et des conventions, et se reprennent souvent entre eux pour se corriger. Cette compétence d'analyse et d'évaluation doit être encouragée et renforcée en classe par un travail d'explicitation des codes et des usages attendus dans telle ou telle situation, ainsi que par un travail visant le développement de la conscience de la dimension symbolique et stratégique du langage (Perrenoud, 1988). Prendre le temps d'une véritable réflexion sur la variation stylistique permet donc à l'enfant de sortir de l'implicite tout un ensemble de variations auxquelles il est confronté quotidiennement et de prendre conscience de ses pratiques ainsi que de celles des locuteurs qui l'entourent, de confronter ses représentations avec celles de ses camarades afin de faire évoluer celles des uns et des autres. Cette prise de distance donne la possibilité aux élèves d'entrer dans les apprentissages de la langue de scolarisation, selon une perspective fonctionnelle.

Pour mener à bien cet objectif, Critchley (1994) propose, dans le cadre de l'enseignement/apprentissage du FLE/2, de travailler à partir d'enregistrements de discours oraux pour mener un travail portant sur les caractéristiques de l'oral et des styles, en axant les analyses sur certaines spécificités du français parlé (les unités de sens remplaçant les phrases, les pauses, faux départ, etc.), sur les marques (syntaxiques, lexicales et phonologiques) pouvant caractériser du discours plutôt formel ou plutôt informel, et sur la notion d'adaptation au contexte. Les activités suggérées s'appuient sur des tâches de transcription soutenues par une grille d'écoute. Or, il pourrait être intéressant d'explorer ce type d'approches dès le cycle 3 en français L1. Travailler sur des extraits d'interactions enregistrées et tenter de les transcrire permettrait d'aiguiser la capacité des élèves à identifier certaines marques saillantes, et d'initier des discussions collectives sur les différentes manières de parler. Les marques ainsi relevées pourraient être reportées dans une grille d'analyse construite avec les élèves et à compléter au fil des écoutes, sans prétention d'exhaustivité, dans le but de rendre conscients et d'affiner les schémas représentationnels préexistants sur les variétés.

En définitive, si la langue de scolarisation tend à être canonisée par l'école, elle n'en reste pas moins un espace de variation au sein duquel les enseignants doivent guider leurs élèves, en particulier ceux pour qui cet espace est peu familier. En cela, la didactique de la variation stylistique a tout à voir avec la didactique du plurilinguisme, comme le suggèrent Castellotti, Coste et al. :

\footnotetext{
"toute langue de scolarisation est plurielle, la langue commune n'est pas une. On peut être 'plurilingue' à l'intérieur et à partir d'une seule langue" (Castellotti, Coste et al., 2008: 14).
}

Les auteurs plaident pour une diversification des expériences scolaires autour des langues et des variétés dans le but d'agir sur les représentations et attitudes des apprenants et de développer leurs capacités langagières, en particulier dans la langue de scolarisation. L'éveil aux styles s'intègre ainsi parfaitement à la dynamique de l'éveil aux langues ${ }^{14}$ dans une perspective d'éducation plurilingue. "Savoir que la variation est constitutive des langues", "savoir comparer des phénomènes langagiers", "savoir tenir compte du répertoire de ses interlocuteurs et des caractéristiques sociolinguistiques pour communiquer efficacement à bon escient" sont ainsi autant de capacités à développer dès l'école primaire (Castellotti, Coste et al., 2008: 20-21).

La formation des enseignants à l'éveil aux styles poursuivrait les mêmes objectifs qu'une formation des enseignants à l'éveil aux langues, qui doit amener les maîtres d'école et professeurs du secondaire

à pratiquer une didactique de la langue plus variationniste, qui ne stigmatise pas les écarts mais tente de les expliquer, de les anticiper même, tout en indiquant nettement les formes légitimes et leurs contextes d'usage. Il convient donc d'utiliser la variation comme point de départ de la réflexion métalinguistique. (Billiez, 2005: 337) 
Certaines ressources existantes telles que Poly-tesse (Programme Janua Linguarum, 2000-2003) proposent ainsi des activités de découverte mêlant des variations inter- et intra-langues dans le but de développer des aptitudes métalinguistiques ayant elles-mêmes la vocation de favoriser les apprentissages langagiers au sens large (Candelier, 2005).

Néanmoins, si l'éveil aux langues peut s'appuyer sur des systèmes souvent standardisés, l'éveil aux styles se heurte à un manque criant de description des variétés: que l'on parle de style standard ou nonstandard, de courant ou de familier, la grammaire des styles n'existe pas, et confère immanquablement à l'enseignement/apprentissage de ces notions un caractère impressionniste.

Peut-on envisager, dans ce cadre, que le style soit objet d'apprentissage, si l'on ne sait pas préalablement définir ni même circonscrire cet objet? Pour travailler sur le style à l'école, il faudra donc sans doute se résoudre à avancer par tâtonnements et petites découvertes, en sachant que les échanges autour des notions seront plus utiles et productifs que les notions elles-mêmes ${ }^{15}$.

La mise en place de jeux de rôles et de jeux d'imitation parodiques peut alors être un bon moyen de susciter des échanges sur la variation stylistique au sein des classes, afin de construire la notion de style en préalable à l'apport d'un métalangage scolaire.

D'autres pistes sont également à explorer, comme l'approche des variations par les "actes de parole périlleux" proposée par Maurer (2001). L'auteur s'appuie sur ce concept décliné en un ensemble de formulations plus ou moins atténuées, plus ou moins directes et plus ou moins agressives constituant un continuum. Il s'agit d'ancrer l'analyse des formes dans une perspective pragmatique tenant compte des intentions communicatives des locuteurs.

Ce travail ne peut se programmer a priori en faisant abstraction des profils scolaires, puisque, chez certains enfants, des représentations bien ancrées et aux résonances identitaires fortes s'avèrent potentiellement "obstruantes" au standard quelle que soit l'approche envisagée. Malgré cela, il nous semble que cette perspective pragmatique présente l'avantage de décomposer l'interaction et de fournir une base pour la réflexion sur la variation stylistique, à partir de micro-situations communicatives facilement appréhendables par les enfants.

Une autre idée, développée par Maurer (2001), qui nous semble intéressante, est d'associer davantage l'enseignement de l'oral et de l'écrit. Ainsi, l'étude de certaines formes grammaticales inscrites au programme peut s'enrichir d'un travail parallèle sur l'usage en contexte de ces formes, à l'oral comme à l'écrit. Par exemple, si l'on construit une séquence sur les formes interrogatives en grammaire, il peut être intéressant d'entrer dans la notion par le biais d'une approche fonctionnelle à l'oral sur le fait, par exemple, de "demander une permission".

Dans une logique comparable, mais en prenant davantage appui sur la littérature de jeunesse que sur le travail sur la langue elle-même, des séquences, au départ uniquement à l'écrit, portant sur le dialogue, le discours rapporté, ou encore la notion de points de vue, peuvent naturellement être enrichies d'un travail réflexif et productif sur la variation à l'oral, par le biais d'activités de mise en scène de dialogues, ou de réécriture de textes avec des positions énonciatives différentes susceptibles de mettre en lumière un certain nombre de variations. L'écriture ou la ré-écriture de dialogues réalistes mettant en scène des interlocuteurs différents dans des situations variées constitue, en effet, une ressource inépuisable pour l'observation réfléchie de la langue dans toute son hétérogénéité, tant sur les variations oral/écrit que sur les variations liées au contexte et aux intentions du locuteur. Les ateliers d'écriture fondés sur le pastiche ou les changements de postures énonciatives sont aussi des activités intéressantes pour pratiquer la variation, à l'oral comme à l'écrit, et organiser des échanges sur les différents moyens linguistiques permettant d'endosser tel ou tel rôle.

Au-delà de ces pistes potentielles de mise en œuvre dans les classes, il nous semble primordial de guider les enseignants en formation dans l'exploration de leurs propres pratiques langagières, afin, d'une part, de les sensibiliser à ce qu'est l'oral et la variation, et, d'autre part, de les amener à s'interroger sur les façons de se positionner en classe, au regard du langage des élèves, de leurs propres attentes et exigences, et de l'image linguistique qu'ils donnent à voir. 


\section{Amener les enseignants à questionner leurs propres représentations et pratiques stylistiques}

L'enseignant n'est plus un "maître à parler" doublé d'un "maître à penser" (Bourdieu, 1982: 32). Lafontaine (1986: 138) le dit même en "liberté surveillée". Malgré tout, il est encore susceptible, au sein de sa classe, de pouvoir "donner le ton" en endossant son rôle de maître, rôle dont les contours sont chaque année à redéfinir en fonction de ce qu'il est, de ce qu'il attend des élèves, et en fonction des élèves eux-mêmes, des groupes-classes, voire des établissements. Réussir à se positionner dans la classe de manière à instaurer une atmosphère de travail qui convienne à tous est un défi en permanence renouvelé. Or, réussir à se positionner "linguistiquement" et stylistiquement fait partie intégrante de cette démarche. Pourtant, cette dimension conserve bien souvent un caractère implicite, fondue dans l'ensemble des paramètres qui caractérisent la relation d'autorité maître/élève.

L'étude de Lafontaine (1986) menée auprès d'enseignants de la région liégeoise a largement contribué à mettre en évidence le système de normes activé lorsqu'ils évaluent des productions orales enfantines, ainsi que leur propre perception de leurs manières de parler. Ainsi, il apparaît que la mission homogénéisante dont se sentent investis les enseignants porte en premier lieu sur le lissage des marques d'appartenance sociale, en parallèle de représentations parfois erronées de ce qu'est la langue légitime ${ }^{16}$ et d'une perception peu objective de leurs propres pratiques. Lafontaine observe que les instituteurs, qui reconnaissent utiliser certaines variantes régionales, ne sont en revanche pas conscients d'utiliser certaines formes qu'ils critiquent pourtant en réception, comme la réalisation "(il) y a" de "il y a", l'usage de la négation simple, ou encore la question formulée sans inversion du sujet.

Le maître est donc supposé incarner le standard, et amener les élèves à le maîtriser, sans que les questions de ce que cela signifie réellement, en particulier à l'oral, et de ce que sont ses pratiques langagières effectives au regard de celles de ses élèves ne soient posées clairement :

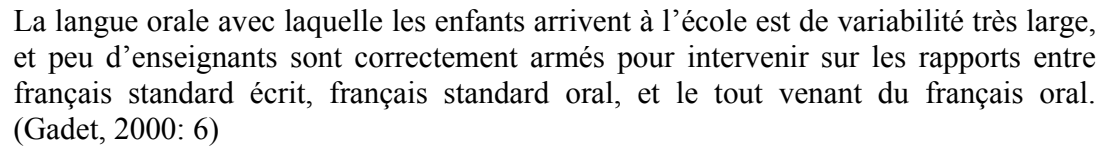

Les erreurs de positionnement sont d'autant plus faciles à commettre dans les établissements défavorisés, où les enseignants sont parfois démunis devant l'ampleur et la difficulté de la tâche, et peuvent être tentés de "s'adapter" à leur public, en proposant des contenus moins ambitieux qu'ils ne l'auraient fait dans d'autres contextes scolaires (voir par exemple Guerin, 2006: 94-95). Pourtant, l'une des ambitions de l'école est de permettre à chacun de construire les connaissances et les compétences nécessaires pour appréhender la complexité du monde. Dans la même optique, la tentation de "s'adapter" au langage des élèves en limitant ses exigences voire en essayant de converger vers certaines pratiques moins standard semble aller à l'encontre de l'enjeu de l'égalité des chances. Parvenir à se situer entre ses propres pratiques, celles des élèves, et les exigences scolaires ne va donc pas de soi, et nécessite sans doute une étape de réflexion menée entre et avec des professionnels.

Une courte enquête menée en 2005 auprès de 32 étudiants de Licence 1 de Sciences du Langage ${ }^{17}$ nous a en outre permis d'approcher certaines représentations de jeunes adultes sur le style. Le document audio que nous avons utilisé est le matériau suivant ${ }^{18}$ :

(1) bon ça - c'est la page d'accueil - hein donc là je n(e) peux rien faire si c(e) n'est - ça - d'accord< - donc à partir de la première connexion - c'est valable deux mois - c'est-àdire - tous ces tickets - on vous les vole $<$ vous nous app(e)lez - nous - on enlève tout $\mathrm{d}(\mathrm{e})$ la base de données - et on $\mathrm{n}^{\prime} / \varnothing$ en- on $\mathrm{n}^{\prime} / \varnothing$ enlève pas ce/ceux qu(e) vous avez vendu - avant $1(\mathrm{e})$ vol $>$ - évidemment $>$ - et nous quandT on vous envoie la facture $<$ on vous envoie le numéro $d(\mathrm{e})$ carnet le numéro $\mathrm{d}(\mathrm{e})$ tiquet le login $<$ donc vous gardez toujours - le coupon et euh la date et l'heure de la première connexion qui correspond pas à la minute près - mais - à l'heure de vente $>$ - pour conclure: $<$ vraiment nous on est euh on est- on connaît bien l'activité d'accès public à l'internet $<$ et euh surtout dans les débuts $<$ n'hésitez pas à nous app(e)ler $>$ 
(2) il est pourri c(e) clavier* - t(u) en a pas un aut(re) là< - et ça $t(e)$ dirait qu'on partage not(re) sandwich $\mathrm{XX}<-$ j'ai unE dalle* - euh tu peux m(e) filer euh un $\mathrm{p}(\mathrm{e}) \mathrm{tit}$ canon d'eau là j'ai soif grave - bon* c'est pa::s un travail d'orfèvre hein* - on n'/Ø est pas dans la microprécision là hein* - ouais bein d'un aut(re) côté j(e) te raconte pas on dirait que: (il) y a eu un braquage* - bon on s'en oufe:: $<*$

Après une écoute, les étudiants devaient répondre par écrit à un questionnaire très court comportant 5 questions ${ }^{19}$. Une analyse qualitative de ces questionnaires a montré que :

- les enquêtés ont d'emblée proposé un commentaire métalinguistique sur les extraits : la dimension métapragmatique devient apparemment saillante chez les récepteurs adultes ;

- les critères évoqués par les étudiants sont très proches de ceux des enfants de 10/11 ans (cf. Buson, 2009), avec des références fréquentes à la norme et à la situation d'interaction (familiarité avec l'interlocuteur, statut, attention portée au discours, "entre soi", reflet d'une image sociale et d'une identité);

- l'influence du parcours scolaire est néanmoins sensible, avec l'usage fréquent du métalangage (familier, courant, soutenu);

- l'école ne semble en revanche pas avoir contribué à diminuer les jugements de valeur sur les variétés : le premier extrait (langage correct, respectueux, courtois, bon usage, bon français, langage précis) est jugé bien plus positivement que le second (vocabulaire populaire, patois, fautes, vocabulaire vulgaire, langage des jeunes ${ }^{20}$ ) alors qu'aucun des deux n'est inadéquat en contexte ;

- la référence de la norme écrite scolaire est omniprésente (référence critique aux phrases courtes à l'oral/longues à l'écrit, aux redondances du sujet "nous on est");

- comme chez les enfants, le lexique (argot, tutoiement) et la prosodie (ton solennel, détendu) semblent les niveaux les plus accessibles à la verbalisation des différences entre les extraits, mais les "ne" de négation et des marqueurs discursifs ont également été relevés. La saillance des marqueurs discursifs considérés comme informels et leur intégration au stéréotype du relâchement est d'ailleurs manifeste, les étudiants relevant parfois des contrastes absents dans les extraits. Par exemple, une enquêtée explique que "dans le premier extrait, on ne trouve pas tous les deux mots des hein et des euh comme dans le second", alors que les deux passages comptent respectivement 1 hein et 3 euh vs 2 hein et 2 euh.

Cette enquête nous a paru éclairante en ce qu'elle montre le peu d'évolutions dans l'appréhension du style à la suite de nombreuses années d'école : à 20 ans et au moment d'entamer leurs études supérieures, les étudiants proposent globalement les mêmes commentaires que des enfants de 10 ans, l'éventail des marques repérées n'est pas plus large et les jugements négatifs à l'égard des variations (tant stylistiques, que sociales, géographiques, ou liées au chenal) sont omniprésents. La seule différence notable est la prégnance de la terminologie, ce qui confirme l'hypothèse selon laquelle il s'agit là de l'unique objectif que les activités proposées à travers les manuels sont susceptibles d'atteindre.

Pour conclure, il nous semble important d'approfondir et d'affiner certaines pistes, comme par exemple :

- la question des niveaux de classe concernés et l'adaptation des activités proposées ;

- la question des modalités d'exploitation des ressources pédagogiques existantes (manuels de FL1 et FLE, Éveil aux langues);

- le travail autour de la transcription de l'oral ;

- l'articulation oral/écrit, avec l'exploitation de ressources de la littérature de jeunesse par exemple ;

- la possibilité d'aborder le style à partir des genres oraux (Dolz \& Schneuwly, 1998) ;

- le potentiel des approches interdisciplinaires intégrant le théâtre21, les jeux de rôles et les improvisations, susceptibles de faire entrer les enfants dans un processus mixte d'exploration d'un potentiel stylistique, par la pratique et la réflexion métapragmatique ;

- le potentiel des approches fonctionnelles intégrant le style et les connaissances grammaticales par les actes de parole (par exemple un travail combiné sur le conditionnel et les variations dans les formulations de requêtes);

- le choix et la conception des méthodes et matériaux déclencheurs permettant d'amorcer une réflexion métapragmatique sur le style ;

- la mise en oeuvre des activités : fréquence, durée des séances, programmation vs progression ou abord des notions au fil de l'eau, évaluation, etc.

Il apparaît en effet tout à fait essentiel de fournir aux enseignants en formation des pistes de réflexion plus complètes sur la variation en général et le style en particulier, afin qu'ils ne soient pas démunis dans leurs classes et qu'ils aient envie de dépasser les approches réductrices proposées dans les livres scolaires. Dans 
cette optique, il convient de réfléchir à des méthodologies et matériaux didactiques concrets pour les sensibiliser au style tout en leur fournissant des outils et des ressources pour construire des séquences en classe.

\section{Références bibliographiques}

Abry, D., Daas, Y., Fert, C., Deschamps, H., Richaud, F. \& Sperandio, C. (2007). Ici. Méthode de français. A2. Paris: Clé International.

Auzanneau, M., Bento, M., Candelier, M., Kervran, M., Ledru-Menot, O. \& Trimaille, C. (2000-2003). Programme Janua Linguarum. Poly-tesse. http://jaling.ecml.at/english/poly-tesse.htm.

Ball, R. (2000). Colloquial French grammar. A practical guide. Oxford: Blackwell.

Berthet, A., Hugot, C., Kizirian, V., Sampsonis, B. \& Waendendries, M. (2006). Alter Ego. Méthode de français. A2. Paris: Hachette Français langue étrangère.

Billiez, J. (2005). Répertoires et parlers plurilingues. Déplacements à opérer et pistes à parcourir pour l'école. In Prudent, L. F., Tupin, F. \& Wharton, S. (Eds.), Du plurilinguisme à l'école. Vers une gestion coordonnée des langues en contextes éducatifs sensibles, Berne: Peter Lang, 323-339.

Blanchet, P. (1995). La pragmatique. D'Austin à Goffman. Paris: Bertrand-Lacoste.

Blanchet, P., Moore, D. \& Asselah-Rahal, S. (2008). Perspectives pour une didactique des langues contextualisée. Paris: Éditions des archives contemporaines. Agence Universitaire de la Francophonie.

Bourdieu, P. (1982). Ce que parler veut dire. L'économie des échanges linguistiques. Paris: Fayard.

Boutet, J. (2002). "I parlent pas comme nous". Pratiques langagières des élèves et pratiques langagières scolaires. VEI enjeux, 130, 163-177.

Boutet, J. \& Gadet, F. (2003). Pour une approche de la variation linguistique. Le français aujourd'hui, 143, 17-24.

Buson, L. (2009). Variation stylistique entre 5 et 11 ans et réseaux de socialisation scolaire : usages, représentations, acquisition et prise en compte éducative. Thèse de doctorat, Université Stendhal, Grenoble.

Candelier, M. (2003). Evlang. L'éveil aux langues à l'école primaire. Bilan d'une innovation européenne. Bruxelles: De Boek-Duculot.

Candelier, M. (2005). L'éveil aux langues. Une approche plurielle des langues et des cultures au service de l'extension des compétences linguistiques. In Prudent, L. F., Tupin, F. \& Wharton, S. (Eds.), Du plurilinguisme à l'école. Vers une gestion coordonnée des langues en contextes éducatifs sensibles, Berne: Peter Lang, 417-436.

Carroll, L. (1961). De l'autre côté du miroir, traduit de l'anglais par J. Papy. Paris: Gallimard.

Carroll, L. (1971). De l'autre côté du miroir et ce qu'Alice y trouva, traduit de l'anglais par H. Parisot. Paris: Flammarion.

Castellotti, V., Coste, D. \& Duverger, J. (2008). Propositions pour une éducation au plurilinguisme en contexte scolaire. Paris: Association pour le Développement de l'Enseignement Bi-/plurilingue.

Charles, F. (1990). Histoire de Lucien. Paris: Rageot.

Coleman, J. A. \& Crawshaw, R. (1994). Discourse variety in contemporary French. Descriptive and pedagogical approaches. Londres: Association for French Language Studies, Centre for Information on Language Teaching. 
Conseil De L' Europe (2000). Cadre européen commun de référence pour les langues. Strasbourg, Paris: Didier.

Critchley, S. (1994). Discourse variety in contemporary French language: a pedagogical approach. In Coleman, J. A. \& Crawshaw, R. (Eds.), Discourse variety in contemporary French. Descriptive and pedagogical approaches, Londres: Association for French Language Studies, Centre for Information on Language Teaching, 203-236.

Dannequin, C. (1977). Les enfants baîllonnés. Paris: Cedic.

Dolz, J. \& Schneuwly, B. (1998). Pour un enseignement de l'oral. Initiation aux genres formels à l'école. Paris: ESF.

Friot, B. (2007 [1992]). Nouvelles histoires pressées. Toulouse: Milan.

Gadet, F. (2000). L'oral : quelles modalités de production pour quelles significations. Extrait des actes du séminaire national "Perspectives actuelles de l'enseignement du Français". Paris, les 23, 24 et 25 octobre. http://eduscol.education.fr/D0033/actfran gadet.pdf. Consulté le 15 septembre 2009.

Gadet, F. \& Tyne, H. (2007). Le style en sociolinguistique : ce que nous apprend l'acquisition. Pratiques, 135-136, 91-99.

Gagné, G. (1983). Norme et enseignement de la langue maternelle. In Bédard, E. \& Maurais, J. (Eds.), La norme linguistique, Québec, Paris: Conseil de la langue française, 463-510.

Grossmann, F. (1996). La mise en texte de la théorie grammaticale dans les manuels de grammaire du primaire et du secondaire. Repères, 14, 57-82.

Guerin, E. (2006). Introduction de la notion de variation situatiolectale dans la grammaire scolaire par la caractérisation de deux opérateurs pragmatiques : "on" et "ça". Thèse de doctorat, Paris X, Nanterre.

Himy, O., Artaud, D., Barjolle, É., Fravega, D., Niel, N., Rappoport, M. \& Vermot-Gauchy, F. (2009). Les Couleurs du Français, Gème. Paris: Hachette Éducation.

Lafontaine, D. (1986). Le parti pris des mots : normes et attitudes linguistiques. Bruxelles: Mardaga.

Liogier, E. (2009). La variation stylistique dans le langage d'adolescents de cité. Langage \& société, 128, 119-140.

M.E.N. (2002). Horaires et programmes de l'enseignement de l'école primaire. B.0. du 14 février 2002.

M.E.N. (2008). Horaires et programmes de l'enseignement de l'école primaire. B.O. du 19 juin 2008.

Maurer, B. (2001). Une didactique de l'oral. Du primaire au lycée. Paris: Bertrand-Lacoste.

Millet, A. (1992). Oral/Écrit : zones de perméabilité. Lidil, 7, pp.113-132.

Mougeon, R., Nadasdi, T. \& Rehner, K. (2002). État de la recherche sur l'appropriation de la variation par les apprenants avancés du FL2 ou FLE. AILE, 17, 7-50.

Oriol-Boyer, C., Duminy-Sauzeau, C., Faure, Y. \& Grappin, D. (1995). Français 5ème. Paris: Hatier.

Perrenoud, P. (1988). "Parle comme il faut !". Réflexions sociologiques sur l'ordre linguistique. In Schoeni, G., Bronckart, J.-P. \& Perrenoud, P. (Eds.), La langue française est-elle gouvernable ? Normes et activités langagières. Neuchâtel et Paris: Delachaux et Niestlé.

Romian, H., Marcellesi, C. \& Treignier, J. (1985). Quelques concepts et notions opératoires pour une pédagogie de la variation langagière. Repères, 67, 23-31.

Todd, Z. (1997). Metaphor, play and drama: the role of the symbolic in the development of sociolinguistic competence. In Thompson, L. (Ed.), Children talking. The development of pragmatic competence, Clevedon, UK ; Philadelphia: Multilingual Matters, 82-90. 
Vargas, C. (1988). Construire la notion de variation langagière. Repères, 76, 1-12.

Verhoeven, L. (2000 [1997]). Sociolinguistics and education. In Coulmas, F. (Ed.), The handbook of sociolinguistics, Oxford, Malden: Blackwell, 389-404.

\footnotetext{
${ }^{1}$ Nous utilisons ici à la fois une terminologie classique en sociolinguistique, celle de style, et une terminologie plus usitée en didactique, celle de registre de langues.

${ }_{3}^{2}$ En référence au courant de l'éveil aux langues (Candelier, 2003 ; Candelier, 2005).

${ }^{3}$ Pour une critique plus détaillée des approches dans d'autres manuels plus anciens, voir par exemple Gagné (1983), Grossmann (1996), ou Boutet \& Gadet (2003).

${ }^{4}$ Sur les équivalences impossibles entre ça et cela, voir Guerin (2006).

${ }^{5}$ Carroll, traduit par J. Papy (1961) et Carroll, traduit par H. Parisot (1971).

${ }^{6}$ Il s'agit du texte de Charles (1990).

${ }^{7}$ Citons à titre d'exemple la nouvelle intitulée Façons de parler de Friot (2007 [1992]) qui met en scène des
} commutations stylistiques dans le cadre d'un dialogue entre un père et son fils. Ce récit humoristique illustre la dimension d'expressivité de certaines formulations : ainsi, le père, affichant au départ un discours à la fois normé et normatif, change radicalement sa manière de parler sous le coup d'une émotion forte.

${ }^{8}$ Cf. Gadet \& Tyne (2007) et Ball (2000) pour des éléments de discussion à propos du bien-fondé de la nécessité de faire apprendre les registres non-standard aux étudiants de FLE/2.

${ }^{9}$ Romian, Marcellesi et al. (1985:29) définissent ainsi le mode de gestion fonctionnaliste plurinormaliste : "il se fonde sur une reconnaissance, une objectivation, une maitrise des codes sociaux, des variations et des normes fonctionnelles qui leur sont liées. Cette approche du code commun, des variations, des normes, de la variabilité est alliée à des comportements pédagogiques qui visent : 1) la diversification, l'objectivation et par conséquent l'appropriation des variations et normes des pratiques langagières, 2) le maniement, la mise à distance, l'analyse, l'intégration des codes sociaux (scolaires et non scolaires)". Voir aussi Vargas (1988).

${ }^{10}$ Voir le CECR, Conseil De L' Europe (2000).

${ }^{11}$ Notons par ailleurs qu'il existe aussi de la variation à l'écrit, ainsi que de multiples "zones de perméabilité" entre l'oral et l'écrit (Millet, 1992), ce qui mériterait également d'être réfléchi et pris en compte à ce niveau.

${ }^{12}$ Cf. Gadet (2000).

${ }^{13}$ Alors que ça paraît nécessaire pour les apprenants de FLE/2, nous ne pensons pas que des enfants de cycle 3 voire de collège aient besoin qu'on leur explique par exemple que "bagnole" est moins standard que "voiture".

${ }^{14}$ Cf., par exemple, Candelier (2003). D'ailleurs, le programme européen dénommé Evlang prévoyait de réaliser un module portant sur la variation diatopique, ce qui ne sera actualisé que plus tard dans le programme Janua Linguarum.

${ }^{15}$ Dans le même ordre d'idées, deux professeures des écoles stagiaires que nous avons encadrées en 2008-2009 pour un mémoire sur l'enseignement/apprentissage de la variation stylistique à l'oral sont arrivées à la conclusion que ce n'était pas lors des jeux de rôles et en position d'acteurs que les enfants pouvaient le mieux faire évoluer leurs représentations. C'était plutôt en se plaçant dans le rôle de l'observateur, en particulier dans les discussions collectives suite aux saynètes les moins pertinentes en termes de variation, que les difficultés des uns à incarner stylistiquement, dans le cadre d'improvisations contrôlées, un rôle social dans une circonstance donnée, permettaient aux autres de construire une discussion collective plus productive.

${ }^{16}$ Le contexte géographique de l'enquête peut toutefois limiter la portée de cette observation: les erreurs de perception de la langue légitime sont souvent liées à l'influence des régionalismes.

${ }^{17}$ Dont la plupart se destinent à l'enseignement.

${ }^{18}$ Il s'agit d'un adulte qui s'est auto-enregistré pendant une journée complète dans différentes situations d'interaction (en rendez-vous professionnels avec des clients et à d'autres moments avec des collègues) à l'aide d'un enregistreur mini-disque avec micro-cravate. Certains extraits stylistiquement contrastés ont ensuite été sélectionnés pour constituer le document audio présenté ici.

${ }^{19}$ Quelles différences remarquez-vous entre les deux extraits ? Comment pourriez-vous décrire et qualifier ces façons de parler? Identifiez et éventuellement classez des éléments de variation que vous avez pu remarquer. Pour chacun des deux extraits, imaginez et décrivez la situation de communication. Comment interprétez-vous ces variations ?

${ }^{20}$ Notons que le locuteur avait 33 ans au moment de l'enregistrement.

${ }^{21}$ Comme le souligne Todd (1997), les activités théâtrales sont importantes pour développer la connaissance des rôles sociaux, à travers la capacité à switcher entre différents rôles et différents points de vue. 